\title{
Phlebologie-Förderungspreis 2018: Erfolgreiche Publikation durch Dr. Luca Spinedi (Locarno, Schweiz) im Journal of Vascular Surgery
}

Luca Spinedi, Hans Stricker, Hak Hong Keo, Daniel Staub, Heiko Uthoff: Feasibility and safety of flush endovenous laser ablation of the great saphenous vein up to the saphenofemoral junction. J Vasc Surg: Venous and Lymphatic Dis 2020. Epub ahead of print

Mit dem wissenschaftlichen Förderungspreis 2018 unterstützte die Schweizerische Gesellschaft für Phlebologie eine Studie zur technischen Durchführbarkeit und Patientensicherheit der endovenösen Thermoablation der V. saphena magna bis an die Mündung („Flush Endovenous Laser Ablation “, analog einer chirurgischen Krossektomie).

In einer prospektiven Patientenserie von 135 Eingriffen bei 113 Patienten, welche wegen einer Krossen- und Stamminsuffizienz der Vena saphena magna mit dem 1470nm-Laser mit radialer Emission der Laserspitze behandelt wurden, wurden die technische Durchführbarkeit, die Erfolgsrate sowie das Auftreten der endovenösen hitzeinduzierten Thrombose (EHIT) untersucht. Die EHIT wird nach Kabnick in 4 Klassen unterteilt: Class $1=$ Thrombose der V. saphena magna bis an die Mündung (hier bei allen Eingriffen das Ziel), Class 2 = partielle tiefe Beinvenenthrombose mit $<50 \%$ Okklusion der V. femoralis communis, Class $3=$ partielle tiefe Beinvenenthrombose mit $>50 \%$ Okklusion der V. femoralis communis, Class 4 = vollständige tiefe Beinvenenthrombose der $\mathrm{V}$. femoralis communis.
Der mittlere Durchmesser der Vena saphena magna an der Krosse betrug 9,4 $\pm 2,7 \mathrm{~mm}$, mit einem maximalen Durchmesser von 16 mm. In 86,6\% (110/135 Eingriffe) wurde kombiniert eine Phlebektomie und in 13,4\% (17/135 Eingriffe) in der gleichen Sitzung eine Schaumsklerotherapie durchgeführt.

In 94,1\% (127/135 Eingriffe) gelang die Laserablation bis an die Magnamündung in die Vena femoralis communis. In 5,9\% (8/ 135 Eingriffe) misslang die exakte Platzierung der Laserspitze an der Mündung in die V. femoralis communis. Bei diesen 8 Eingriffen wurde die endovenöse Laserablation in der üblichen Technik mit 10$20 \mathrm{~mm}$ Abstand von der Mündung ausgeführt.

Endovenöse hitzeinduzierte Thrombosen (EHIT) traten in 1,6\% (2/127 Eingriffe) auf, 1-mal eine EHIT Class 2 und 1-mal eine Class 3. Die EHIT Class 3 wurde am 10. postinterventionellen Tag diagnostiziert. Unter einer therapeutischen Antikoagulation mit Rivaroxaban lösten sich die EHIT bei beiden Patienten innerhalb von 3 Wochen duplexsonografisch vollständig auf.

Bei 1 Patienten entwickelte sich eine superfizielle Venenthrombose und bei 1 weiteren Patienten eine tiefe Unterschenkelvenenthrombose an der Stelle einer durchgeführten Phlebektomie. In der Leiste wurden keinerlei Komplikationen oder trophische Störungen beobachtet.
Der exakte Verschluss bis auf das Mündungsniveau der Vena saphena magna war in der Duplexsonografie am 1. postoperativen Tag in 94,5\%, nach 10 Tagen in 95,3\% und nach 6 Wochen in 88,2\% der durchgeführten Eingriffe immer noch nachweisbar.

Schlussfolgerung: Grundsätzlich ist die endovenöse Laserablation $(1470 \mathrm{~nm}$, radiale Emission) bis auf das Mündungsniveau in die $\mathrm{V}$. femoralis communis in rund $95 \%$ der Eingriffe technisch realisierbar. Nach 6 Wochen hatte sich der Thrombus bei rund $12 \%$ der erfolgreich behandelten Patienten leicht zurückgezogen, während der Verschluss bei den verbleibenden $88 \%$ immer noch bis an das Mündungsniveau reichte. EHITs traten bei 1,6\% der Eingriffe auf und lösten sich unter einer therapeutischen Antikoagulation innert 3 Wochen vollständig auf. Somit ist die Komplikationsrate der endovenösen Laserablation der V. saphena magna bis auf das Mündungsniveau in die V. femoralis communis („Flush Endovenous Laser Ablation“) in dieser ersten größeren prospektiven Patientenserie nicht höher als bei der bisher üblichen Technik, welche 10$20 \mathrm{~mm}$ vor der Mündung ins tiefe Beinvenensystem stoppt.

Wie immer sollten solche vielversprechenden Resultate einer größeren Fallserie in einem nächsten Schritt in einer randomisierten kontrollierten Studie überprüft werden. 